\title{
EN TORNO A LA DISCUSIÓN SOBRE «DESINDUSTRIALIZACIÓN» Un sucinto análisis respecto a la producción bruta y el Producto Interno Bruto
}

\author{
Julio Silva-Colmenares ${ }^{1}$
}

\section{RESUMEN}

Este artículo presenta las reflexiones del autor sobre lo que ha venido llamándose la «desindustrialización», comenzando por la duda si la utilización del prefijo des es la mejor opción gramatical para su denominación. También muestra que tampoco corresponde a un proceso, en general, de pérdida de importancia de la industria o, peor aún, de su desaparición, sino de la disminución de su peso relativo en algunas variables macroeconómicas, lo que es un fenómeno diferente. Pérdida de peso relativo que responde a transformaciones sustanciales en la «cadena de producción», sin que disminuya, en términos absolutos, el volumen y el valor de la producción industrial. Como ejemplo se toma la industria colombiana respecto a la producción bruta y el Producto Interno Bruto en el lapso 1965-2010. En el caso colombiano, no deja de ser preocupante lo ocurrido, por lo que además de analizar el efecto de tales cambios en la medición, habría que investigar sobre su significado en el largo plazo. Mientras tanto, crecen a mayor ritmo $y$, por consiguiente, aumentan su peso relativo otros sectores, dentro de los cuales se encuentran aquellos en donde se «tercerizan» bienes y servicios que se incorporan a la producción industrial.

Palabras clave: industrialización, desindustrialización, cadena de producción, crecimiento desigual, medición económica.

Clasificación JEL: E23, L60, O14.

1. Fundador, miembro de número, coordinador de la Comisión sobre Problemas del Desarrollo y Vicepresidente de la Academia Colombiana de Ciencias Económicas; miembro correspondiente de la Real Academia de Ciencias Morales y Políticas de España; PhD en economía (summa cum laude) de la Escuela Superior de Economía de Berlín y doctor en ciencias económicas de la Universidad de Rostock (Alemania); director del Observatorio sobre Desarrollo Humano y profesor-investigador emérito de la Universidad Autónoma de Colombia; presidente de la Fundación América Mundial; profesor visitante de postgrado en varias universidades, sobre todo en la temática del desarrollo humano y la gerencia social; autor de 13 libros, 21 folletos y más de 300 ensayos y artículos científicos publicados en Colombia y el exterior, de los cuales más de 70 en revistas indexadas o de importancia académica; coautor en 23 libros. obdehumano@fuac.edu.co

Fecha de recepción: 10 de abril de 2014 - Fecha de aprobación definitiva: 30 de mayo de 2014 


\title{
AROUND THE DISCUSSION ON «DEINDUSTRIALIZATION» A brief analyzes about the gross production and the Gross Domestic Product
}

\author{
Julio Silva-Colmenares
}

\begin{abstract}
This article shows the author's reflections on what has been called «deindustrialization", starting with the question whether the grammatical use of the prefix de is the best choice for its denomination. It also reveals that neither corresponds to a process of decreasing importance of the industry nor much less its disappearance, but rather a decline in their relative weight in some macroeconomic variables, which is a different phenomenon. A less relative weight that respond to a substantial changes in the "production chain», without decreasing in absolute terms, both in volume and value of industrial production. As an example it is taken the gross output and GDP from Colombian industry in the period 1965-2010. The Colombian case exhibits a worrying situation that implies not only an analysis about the effect of such changes in the measurement, but also it is compulsory to investigate its significance in the long term. Meanwhile, other sectors are growing faster and therefore they increase their relative weight, some of them are among those where goods and services are «outsourced» and then they are incorporated into industrial production.
\end{abstract}

Key words: industrialization, deindustrialization, production chain, unequal growth, economic measurement.

JEL Classification: E23, L60, 014. 


\section{UNA PARADOJA CON PREGUNTAS OBVIAS Y UN INTERROGANTE SOBRE EL USO DEL PREFIJO DES}

En la actualidad se presenta una situación paradójica: a medida que en distintas partes y en momentos diferentes -en el trabajo, en el hogar, en el deporte, en el transporte, en los momentos de ocio, con un largo etcétera, o en la mañana, la tarde, o la noche- se utilizan más bienes de origen industrial, crece la idea de que está ocurriendo una creciente desindustrialización. Ante esta realidad, surgen unas preguntas obvias: ¿De dónde, entonces, provienen todos esos bienes que nos abruman en nuestra vida cotidiana? ¿Si cada día hay más y más bienes industriales, y se «industrializan» muchas actividades cotidianas -desde cocinar en los hogares hasta enseñar en colegios y universidades, para mencionar algunas-, porqué se habla de desindustrialización? Y Colombia no escapa a esa situación, por lo que en los últimos años se ha intensificado la discusión sobre si ha ocurrido tal proceso, para lo cual se utiliza con frecuencia como indicador básico el peso relativo de la industria manufacturera en el Producto Interno Bruto -PIB- del país. O la ocupación directa en la industria manufacturera.

Como ejemplo podemos citar lo que dice al respecto un documento de ANIF, reconocido centro colombiano privado de pensamiento económico. En la Introducción se afirma que "Colombia ha venido experimentando un proceso de desindustrialización a través del cual su relación Valor Agregado Industrial/PIB ha venido descendiendo de niveles del 24\% hace tres décadas a uno del 15\% hace una década y actualmente se perfila hacia tan sólo 9\%-12\% en dicha relación hacia el período 2012-2020. En términos de generación de empleo, la industria aportaba cerca del $25 \%$ del total de empleo hace tres décadas, hace una década aportaba el $23 \%$, pero actualmente sólo contribuye con el 13\%”. Idea que reiteran en las Conclusiones (Clavijo, Vera y Fandiño, 2012: 11-77).

Y la respuesta a esta paradoja no puede ser ajena al interés investigativo de nuestro Observatorio sobre Desarrollo Humano ${ }^{2}$, ya que vista la producción industrial por el lado de la oferta significa población ocupada y, por ende, el ingreso monetario o remuneración, ya sea directo o indirecto, de miles de personas. La oferta de bienes y servicios es producto exclusivo del esfuerzo creador de los trabajadores y no tanto del capital y la tierra, que son apenas factores inanimados que coadyuvan a dar forma a esa oferta. Y por el lado de la demanda se traduce en riqueza social, tangible en bienes e intangible en servicios, que, en su mayor parte, consumen todos los hogares, además de los bienes que están destinados al consumo de la administración pública, las demás empresas y la exportación. Es decir, tiene una relación estrecha con el bien-estar ${ }^{3}$ o búsqueda de la felicidad,

2. Para información sobre el Observatorio, véase su sitio en la página de internet de la Universidad Autónoma de Colombia en el siguiente enlace: http://www.fuac.edu.co/index.php?mod =12

3. No usamos la palabra bienestar, unida, como es lo usual, sino separada por un guión para resaltar que el bien-estar no es tanto un disfrute de bienes materiales -como es la interpretación deformada que tienen algunas corrientes del pensamiento-, sino el conjunto de aspectos materiales y espirituales que permiten al ser humano estar bien, realizarse con satisfacción, con independencia de lo material, sin que se niegue su necesidad. Esta licencia la llama el lexicógrafo español José Martínez de Sousa el "guión estilístico o geminado", que es "el que suele colocarse, en un término 
así no sea su componente principal. Como hipótesis de partida para estas líneas, se considera que para resolver la paradoja hay que cambiar la forma de medir la actividad económica y la creación de riqueza, pues como se ha venido haciendo no tiene en cuenta los cambios sustanciales que han ocurrido en la utilización del conocimiento, el uso de los recursos y del propio esfuerzo humano en el proceso de trabajo, como se trata de indicar en distintas partes de esta nota.

Pero antes de seguir adelante, habría que señalar que una cosa es que la industria haya perdido peso relativo en el total del PIB, como se desprende del documento de ANIF, y otra cuestión distinta es que haya perdido importancia, como puede dar a entender la palabra desindustrialización. Adelantándonos un poco al análisis que presenta esta nota, en la tabla 1 puede verse que la producción bruta -PB-industrial, en precios constantes de 1994, aumentó de 8,6 billones en 1965, a 20 billones en 1978, a 27,7 billones en 1990 y 53,7 billones en 2010, años que hemos tomado como hitos en nuestro análisis, por razones explicadas en las páginas siguientes; a su vez, el PIB industrial aumentó de casi 3 billones en 1965, a 7,8 billones en 1978, a 11,3 billones en 1990 y casi 14 billones en 2010; es decir, en los 45 años, la PB aumentó a una tasa anual acumulativa de 4,15\% y el PIB del 3,51\%, que si bien son guarismos inferiores a los observados en algunos países asiáticos, para nuestra realidad y el entorno latinoamericano no son tan mediocres. Además, son tasas superiores al incremento de la población, lo que implica un crecimiento que sobrepasa las necesidades mínimas del crecimiento demográfico.

Visto así el fenómeno ocurrido -y no sólo en Colombia, sino en muchos países del mundo- creemos que se está utilizando con un significado equivocado la categoría desindustrialización, palabra compuesta resultante de anteponer el prefijo des al sustantivo industrialización. Descompongamos este argumento; de industrialización dice el Diccionario de las academias de la lengua española que es la "Acción y efecto de industrializar", y de industrializar dice, en su primera acepción, que es "Hacer que algo sea objeto de industria o elaboración". Y la industria la define, en su acepción 2, como el "Conjunto de operaciones materiales ejecutadas para la obtención, transformación o transporte de uno o varios productos naturales".

A su vez, la Nueva Gramática de las Academias de la lengua española dice respecto al prefijo des que "Con este prefijo se crean numerosos nombres derivados que denotan acciones, situaciones o propiedades opuestas a las expresadas por sus bases nominales, como desacuerdo, desdicha, deshonor, despropósito o desvergüen$z a$. En otros sustantivos, el prefijo se interpreta con sentido privativo: desaseo, desempleo, desmesura, desobediencia, desorden. (...)". Y más adelante señala que "Se distinguen cuatro interpretaciones de des- en los derivados verbales: 1) Ausencia (desconocer algo 'no conocerlo'); 2) Cese, cancelación o anulación (deshabitar un

compuesto, para separar sus elementos cuando conviene hacer hincapié en una de las acepciones de tal compuesto...”. Ortografía y ortotipografía del español actual. $2^{\mathrm{a}}$ edición, corregida. Ediciones Trea, Gijón, 2008. pp. 361-362. El Diccionario de las academias de la lengua destaca que la palabra bienestar viene de bien y estar. En inglés también se está haciendo esta distinción, pero ha sido más fácil: Se ha sustituido walfare por well-being. En francés se está utilizando la palabra compuesta bien-être. 
lugar 'dejar de habitarlo'); 3) Acción contraria (descoser un vestido 'desprenderle las puntadas'); 4) Acción inadecuada (desinformar a la gente 'informarla mal')”4 .

En el caso del fenómeno que nos interesa, la categoría desindustrialización no puede significar lo opuesto a industrialización, o tener un sentido privativo, como los ejemplos que muestra la Nueva Gramática (despropósito, desempleo, etc.), o la interpretación de ausencia, anulación, acción contraria o acción inadecuada, pues la industria no ha desaparecido ni está en proceso de desaparecer, ya que lo que ocurre es que está cambiando de contenido o incluso de esencia, dadas las transformaciones sustanciales por las que pasa, como lo veremos más adelante. Estos cambios la han llevado a perder peso relativo en el agregado nacional, sin que haya perdido importancia.

Incluso mejorías significativas en la actividad industrial, como por ejemplo la elevación de la productividad (más unidades de producto por unidad de tiempo) o innovaciones en proceso y materiales (como la sustitución de minerales tradicionales pesados por nuevas aleaciones livianas), pueden llevar a reducciones importantes en costos unitarios, lo que hace que disminuya el monto dinerario y aumente el volumen producido, afectándose su peso relativo en el agregado nacional ${ }^{5}$. Hoy se habla de una «tercera revolución industrial», lo que implica una transformación sustancial en la actividad industrial ${ }^{6}$. Nueva producción que supone la sustitución de la fábrica de antaño, movida por el motor de vapor y la electricidad, con mucho trabajo humano «presencial y sencillo», por la empresa de hogaño, con mucha ciencia acumulada, que se mueve por impulsos electrónicos, y que utiliza poco trabajo humano «vivo» pero de alta especialización. El gran reto de hoy es buscarles empleo a los trabajadores que ya no pueden ocuparse en las actividades «desaparecidas», lo que no es propósito de estas notas.

Alrededor de estas ideas nos moveremos en las páginas siguientes, orientados por el convencimiento de que es más un problema de medición que de otra cuestión, como para otros aspectos lo identificó la Comisión Sarkozy, según lo veremos

4 ASOCIACIÓN DE ACADEMIAS DE LA LENGUA ESPAÑOLA. Nueva gramática de la lengua española. Manual. Bogotá: Editorial Planeta Colombiana, 2010, pp. 187-188.

5. Un investigador estadounidense mostró que "una porción sustancial de la caída en el empleo industrial en EE.UU. se debe a la caída dramática en el número de empleados durante las últimas décadas en un grupo pequeño de plantas gigantescas cuya producción por otro lado nunca disminuyó. Él le atribuye esta caída a la automatización de las plantas y a la tercerización de actividades no esenciales", En: CARRANZA, Juan Esteban y MORENO Stefany. Tamaño y estructura vertical de la cadena de producción industrial colombiana desde 1990. Borradores de Economía: Banco de la República. 2013. No. 751, p. 4.

6. Según la revista The Economist -citada en el trabajo de ANIF-, "Ahora una tercera revolución industrial está en camino. La manufactura se ha vuelto digital (...), lo cual bien podría cambiar no sólo el curso de los negocios, sino muchas cosas más (...), pues las tecnologías están convergiendo: software inteligente, materiales novedosos, robots de gran destreza y la impresión en 3D permite fabricar prototipos casi de cualquier cosa. (...) La geografía de la cadena de abastecimientos cambiará (...) Como todas las revoluciones esta será perturbadora. (...) La mayoría de los trabajos ya no estará en las fábricas propiamente dichas, sino en las vecinas oficinas de diseño, llenas de ingenieros de alta tecnología, expertos en logística y comercializadores (...), afectando no sólo cómo se hacen las cosas, sino dónde (...)”. CLAVIJO, Sergio; VERA Alejandro y FANDIÑO Alejandro. Op. cit., p. 17. 
más adelante. Esto no niega que en el caso de Colombia haya que tener en cuenta aspectos colaterales que pueden haber disminuido la producción industrial, como el hecho de que muchas empresas industriales se han convertido en importadoras de bienes terminados -como ha ocurrido, por ejemplo, en las ramas de confecciones y productos químicos o en la industria automotriz y de máquinas-herramientaso que muchas que producían en Colombia para exportar, ahora son productoras en el exterior, en un creciente proceso de «transnacionalización» del gran capital colombiano (Silva-Colmenares y Padilla Pardo, 2013: 29-57). Habría que estudiar con más detalle fenómenos propios de «deformación» en la estructura industrial en capitalismos «tardíos»y de «monopolización precoz», como el colombiano.

\section{DOS FORMAS DISTINTAS DE CUANTIFICAR LA ACTIVIDAD ECONÓMICA}

Volviendo al análisis del fenómeno, puede decirse que la forma más tradicional de cuantificar la actividad económica ha sido a través de la composición sectorial del Producto Interno Bruto -PIB-, uno de los resultados principales del Sistema de Cuentas Nacionales, con base en las matrices de insumo-producto. Para evitar la doble contabilización que se produciría cuando un bien o servicio pasa de un sector a otro, para una mayor elaboración o transformación, el PIB de cada sector es, en la práctica, la sumatoria de los valores agregados -VA- en ese sector. Como es natural, algo diferente ocurre con el resultado neto del comercio exterior (exportaciones menos importaciones), lo que también afecta al PIB de manera indirecta. $\mathrm{Al}$ ser en lo fundamental el valor neto de la actividad económica durante un lapso determinado (por ejemplo, un año), tiene la ventaja de representar la riqueza neta creada por esa sociedad durante ese período, ya sea en forma tangible o intangible.

Otra forma de cuantificar la actividad económica, aunque menos usual pero factible, es también a través de la composición sectorial pero de la Producción Bruta -PB-, esto es, incluido el consumo intermedio -CI-, o sean los bienes o servicios que pasan de un sector para otro, con distintas finalidades. Esta modalidad tiene la desventaja de las doble, triple, o poli contabilizaciones, pero tiene la ventaja que permite apreciar la verdadera magnitud de la actividad económica de cada sector, pues corresponde a los precios finales de ese sector. Como los sectores tienen muy diferente CI, dado el nivel de transformación que cada uno contempla, muy diferente será su peso relativo, ya sea como componente del PIB o de la PB. Por tanto, el propósito de este documento es proponer que se deben presentar y analizar, siempre que sea posible y mientras se «produce» por parte de las autoridades correspondientes un método alternativo de medición, estas dos macro variables, pues mientras el PIB mostraría, en resumen, la riqueza neta creada durante un determinado lapso, la PB mostraría la magnitud de la actividad económica, incluida la utilización de riqueza creada antes, esto es, el CI.

En la tabla 1 sección A (que se encuentra al final) se observa de manera nítida esa situación para el lapso 1965-2010, pues en el caso de la industria manufacturera -la que interesa en primer lugar para esta nota-, su peso relativo en el PIB disminuyó del 19\% en 1965 (el año más antiguo sobre el cual se pudo conseguir 
información comparable con la actual) al 12,8\% en $2010^{7}$, esto es, perdió 6,2 puntos porcentuales, casi una tercera parte de su peso relativo, el $32,7 \%$ en términos exactos. En la PB perdió un poco menos, el 30,3\% de su peso relativo, y disminuyó del $34,4 \%$ al $24 \%$ en el mismo lapso; pero todavía la producción industrial sigue aportando casi una cuarta parte de toda la actividad económica y es el sector con mayor contribución, si se le mide por la PB. En cambio, el sector de los servicios a las empresas y las personas, incluido el gobierno y el servicio doméstico, aumentó su participación en el PIB del 11,6\% al 15,8\% en el mismo lapso, o sea 4,2 puntos porcentuales, casi el 37\%; es de observar que en este sector se generan ahora bienes y servicios que antes se producían en el interior de las empresas agropecuarias e industriales, en un proceso creciente de lo que se ha llamado la «terciarización» o suministro por «outsourcing», según la expresión en inglés. Lo mismo puede estar ocurriendo con el sector de comercio, reparaciones, hoteles y restaurantes, así como en transporte, almacenamiento y comunicaciones, aunque los dos perdieron participación en el PIB, pero la ganaron en la PB.

Como se aprecia en la tabla 1 sección A, para 1965 los dos sectores con mayor peso relativo en el PIB eran el agropecuario con más del 27\% y la industria manufacturera con el 19\%, lo que sumaba más del 46\%, seguidos por servicios financieros y de alquiler de vivienda con casi 15\% y comercio, reparaciones, hoteles y restaurantes con un poco más de 13\%. Cuarenta y cinco años después los dos de mayor peso relativo en el PIB son servicios financieros y de alquiler de vivienda, con más del 19\% y servicios personales, a las empresas, de gobierno y doméstico, con casi 16\%, para totalizar el 35\%; la industria ocupa el tercer puesto con casi 13\% y en el cuarto lugar va comercio, reparaciones, hoteles y restaurantes con cerca del 12\%; es evidente la importancia que han adquirido los servicios, aunque en muchas de sus empresas los servicios se prestan a través de la producción de accesorios o suministros materiales, lo que permitiría hablar también de producción «fabril» o «industrial» en estos sectores.

Con base en la misma tabla 1 sección A, pero visto el «ranking» en la PB, para 1965 el primer lugar lo ocupaba la industria manufacturera, con el 34,4\%, seguida por el sector agropecuario, con el 19,4\%. Les seguían los servicios personales, a las empresas, de gobierno y doméstico y el sector financiero e inmobiliario, con casi el 23\%. Cuarenta y cinco años después la industria sigue teniendo el mayor peso relativo, con el 24\%, lo que corrobora la importancia del CI en la industria, pero el sector agropecuario pierde el segundo puesto, en beneficio de los servicios personales, a las empresas, de gobierno y doméstico, con el 21,4\%, y el comercio, reparaciones, hoteles y restaurantes ocupa el cuarto puesto, con el 12,6\%. Como en el caso del PIB, también en la PB ganan peso relativo sectores hacia donde se

7 Para la elaboración de las tablas, además de los años 1965 y 2010, se tomaron 1978 y 1990, pues son los años finales de los dos primeros sub-períodos que abarca el proyecto de investigación Colombia 1959-2010: Principales transformaciones económicas, sociales y políticas, que adelanta el Observatorio sobre Desarrollo Humano, con participación de estudiantes graduandos como investigadores auxiliares. La información primaria para esta nota fue recopilada por los graduandos Dewar Hurtado y Liz Polanía, del programa de Economía de la Universidad Autónoma de Colombia, quienes adelantan un proyecto específico de grado sobre cambios en la población ocupada durante el mismo lapso. 
supone se han desplazado actividades que antes se hacían en empresas típicas de la manufactura o de la labor agro-industrial.

O sea, habría que investigar más a fondo que actividades de «servicios», como reparaciones, aseo, contabilidad, ventas, transporte, almacenamiento, servicios a los trabajadores y a los clientes y similares, ya no se realizan con personal de las empresas manufactureras o agro-industriales, es decir, ya no se contabilizan como parte de su valor agregado, por no corresponder a su objeto social principal, y ahora se «producen» en empresas especializadas de otros sectores, como los mencionados en las líneas anteriores, las que también pueden producir algún tipo de bienes materiales. Empresas de servicios, que incluso pueden ser de los mismos propietarios de las empresas fabriles de la ciudad y el campo, dado el creciente surgimiento de conglomerados empresariales en Colombia que «encadenan» el proceso de producción y distribución en sistemas de monopolio vertical y horizontal. Estos servicios «encadenados» pueden explicar, si no todo, por lo menos parte del peso relativo que perdió la actividad agropecuaria y la industrial. Es decir, son «antiguos» trabajos «industriales» que ahora se realizan como «nuevos» en otros sectores.

Pero este proceso no podría llevarnos a hablar de desindustrialización, en el sentido prístino de esta categoría, sino de disminución de su peso relativo por transferencia de labores a otros sectores, como lo señala muy bien The Economist al hablar de la «tercera revolución industrial». Por eso creemos que a este proceso no se le podría denominar de «desindustrialización secular», como lo llama el documento de Clavijo, Vera y Fandiño (2012:49) siguiendo la propuesta de Rowthorn y Ramaswamy, pues si bien ha habido "transferencia de trabajo del sector industrial al sector servicios" y ha ocurrido una "expansión del sector terciario", ello no ha llevado a la disminución absoluta de la producción industrial, menos si pudiéramos adicionar los bienes y servicios que antes se producían en el sector industrial y ahora se elaboran en otros sectores. Los investigadores de ANIF también explican lo que llaman desindustrialización como un efecto eventual de la "posible» enfermedad holandesa padecida por Colombia en los últimos lustros, aunque a este efecto también podría aplicarse el razonamiento anterior. No obstante, Clavijo, Vera y Fandiño aclaran que "las hipótesis de una desindustrialización «secular» explicadas por la expansión del sector de servicios (...) y por el nivel de desarrollo (...) no tenían asidero econométrico para el caso de Colombia. Estos resultados implican que la teoría de la EH [enfermedad holandesa] (Corden y Nearly, 1984) (...) es la que mejor explica la «prematura» desindustrialización que ha experimentado Colombia durante los últimos cuarenta años".

En las Conclusiones de su trabajo Clavijo, Vera y Fandiño (2012: 61, 77, 78) constatan -son sus palabras- "un acelerado proceso de desindustrialización durante 1965-2012” [período que coincide con el lapso que analizamos en este documento] asociado a: "i) serias dificultades estructurales en la provisión de los servicios más básicos (energía, telecomunicaciones, vías de transporte); y ii) el efecto del auge minero-energético, acompañado de encarecimiento relativo de la mano de obra y marcada apreciación real de la tasa de cambio, confirmando la hipótesis de la Enfermedad Holandesa" Aunque no es motivo de discusión en este documento, al contrario de lo que podría pensarse según lo dicho en las líneas anteriores por los tres investigadores de ANIF, en la tabla 2 se observan tasas anuales acumulativas 
per cápita de crecimiento del CI y el VA en los sectores de electricidad, gas y agua y de construcción y obras públicas de magnitudes por encima del promedio, al tiempo que hay estudios que hablan más bien de una precarización del mercado laboral que de encarecimiento de la mano de obra (Ferreira, 2010: 193).

Una idea similar de desindustrialización se encuentra en el documento $E l$ proceso colombiano de desindustrialización, de Juan José Echavarría y Mauricio Villamizar (2006, 53-54) pero que en realidad cubre una temática más amplia, pues analiza el proceso de industrialización durante varios períodos históricos, con aportes muy importantes. Para resumir su idea sobre lo que nos interesa, en las Conclusiones leemos: "Nuestro proceso de industrialización tuvo corta duración. La industria colombiana ha disminuido su participación en el empleo total desde los 1960s (sic) y en la producción desde mediados de los 1970s (sic). El fenómeno de desindustrialización se ha dado con especial fuerza en la industria moderna. Se trata del efecto conjunto de dinámicas mundiales (aún no satisfactoriamente analizadas en la literatura) y de la política económica”. Aunque en el párrafo siguiente se dice algo que parece contradecir lo anterior: "(...) y la evidencia empírica confirma que hoy se cuenta con un sector industrial moderno mucho más competitivo, preparado para afrontar el reto de la globalización. Las exportaciones se han convertido en el centro del crecimiento, la productividad multifactorial creció nuevamente en los 1990s (sic), reversando la tendencia observada en los 1980s (sic), y el valor agregado por trabajador ha crecido a tasas incluso superiores a las de los Estados Unidos" (Echavarría y Villamizar, 2006: 53-54).

Un documento de dos investigadores del Banco de la República coincide más con la apreciación nuestra e incorpora una tercera forma de medición. Según sus palabras textuales, "A diferencia de los estudios usuales sobre el tamaño de la industria que se basan en medidas del tamaño relativo del valor agregado de las firmas industriales, nuestras medidas del tamaño de la industria se basan en el valor agregado de toda la cadena de producción industrial. (...) Para ser más específicos: el valor agregado industrial incluye el valor agregado de todos los insumos que se producen al interior de una firma, incluso aquellos que no son propiamente industriales como el aseo, la vigilancia, el mantenimiento, etc. Si una firma industrial «terceriza» la producción de estos insumos, su valor agregado disminuye, pero el valor agregado de la cadena de producción industrial no cambia. A diferencia del valor agregado, nuestras medidas del tamaño de la cadena de producción industrial incorporan consistentemente todos los insumos industriales, independientemente de si se producen o no en el interior de las firmas".

Más adelante dicen: "El cambio en el tamaño relativo del valor agregado de la industria y sus actividades conexas es un fenómeno contable, pero tiene raíces económicas. Estas raíces son los incentivos sistemáticos de las firmas a integrar o desintegrar sus cadenas de producción, que conllevan cambios en la porción de actividades productivas que se contratan al interior de las firmas o a través del mercado. Estos cambios son responsables en alguna medida de las transformaciones en el tamaño relativo de la industria en todos los países y han sido poco estudiados empíricamente". Y ya para concluir el trabajo dicen: "La desintegración vertical de las cadenas productivas ha tenido un efecto sobre el tamaño relativo del valor agregado sectorial. Esta observación es relevante más allá del caso colombiano 
y abre una interesante agenda de investigación. Por ejemplo, es posible que una parte significativa de la caída en el empleo industrial que se observa en el mundo desarrollado está explicada por la «tercerización» sistemática de servicios a la industria durante las últimas décadas".

En cuanto a lo que es más pertinente para nuestra nota, el documento señala que "La noción de una posible «desindustrialización» de la economía colombiana se basa en los datos de participación del valor agregado industrial en el PIB que (...) ha pasado de ser el 23\% del PIB en 1980 a ser el 13\% del PIB en 2010. (...) Ahora bien, la posible caída en la participación del valor agregado industrial en el PIB no significa necesariamente que este haya caído: significa sencillamente que ha crecido menos que el resto de la economía. Esto, sin embargo, no quiere decir mucho sobre el tamaño relativo de la industria, pues como ya argumentamos el valor agregado es una medida poco satisfactoria de este. Tal como indicamos en la sección 3, una medida consistente del tamaño de la industria debe estar basada en el tamaño de toda la cadena de producción (...)".

Según estos investigadores, "la producción industrial a la puerta de la fábrica como proporción de la producción bruta de la economía ha pasado del 32\% en 1990 a $24 \%$ en 2010 [nota de JS-C: cifras que coinciden bastante con las que se muestran en la sección A de la tabla 1 de nuestro trabajo]. Si se tiene en cuenta los márgenes de comercialización y transporte, el valor bruto de la cadena industrial como proporción de la producción nacional bruta ha pasado de representar el 40\% al 31\%. De nuevo, el grueso de la caída de esta medida del tamaño relativo de la cadena industrial se dio durante la década de los noventa y ha permanecido más o menos constante durante la última década”. Como destaca la Conclusión de este trabajo, "actualmente la cadena de producción industrial colombiana absorbe alrededor del 35\% de la actividad económica. Notablemente, no existe ninguna evidencia de que la actividad industrial haya perdido importancia en la economía durante la última década y mucho menos evidencia hay de que su tamaño se haya reducido" (Carranza y Moreno, 2013: 1-18).

Pero más crítico que la situación de la industria es el resultado que muestra el sector agropecuario, pues disminuyó, en términos generales, en tres cuartas partes su peso relativo en el PIB y en la PB. En cambio, la minería, la producción de electricidad, gas y agua y la construcción y obras públicas aumentaron de manera significativa su participación en el PIB y en la PB, como se puede ver en la tabla 1 sección A, últimas dos columnas. Como comprobación de ese cambio de peso relativo, mientras en 1965 los cinco sectores representativos de la llamada producción material o «economía real», como la llaman otros (renglón 6 de la tabla 1 sección A) representaban casi el 55\% del PIB, para 2010 habían disminuido al 38,5\%, mientras los servicios (renglón 12 de la tabla 1 sección A) incrementaron su participación de un poco más del $45 \%$ al 61,5\%. La disminución de tal participación la explica, en lo fundamental, el sector agropecuario, lo que indicaría que ha ocurrido más bien un grave y culposo retroceso agropecuario, dadas las ventajas naturales que tiene el país para haber aprovechado mejor durante la segunda parte del siglo 20 y principios del siglo 21 la creciente demanda por bienes provenientes de ese sector por parte de muchos países en proceso de crecimiento y desarrollo. Sin negar que la industria atraviese una situación crítica, que debe solucionarse replanteando 
su futuro, es urgente estudiar a fondo lo que ocurre en el campo. Es posible que la solución pase por una política integral agro-industrial que «encadene» más estas dos actividades básicas de nuestra sociedad.

En la sección B de la tabla 1 pueden verse esos cambios en la composición de la PB y el PIB, pero en cifras absolutas, expresadas en pesos constantes de 1994. Como es natural, en un lapso de mediano plazo como el estudiado (45 años), cuando el Sistema de Cuentas Nacionales ha cambiado de base varias veces, mantener la «comparabilidad» de las cifras en el tiempo, implica casi siempre sacrificar algo de la exactitud en beneficio de la homogeneidad, para poder continuar las series estadísticas, por lo que los valores indicados puede que no coincidan con los presentados en otras investigaciones. Incluso Carranza y Moreno recuerdan que las series de Cuentas Nacionales con base en los años 1975, 1994 y 2005 "no son comparables pues se refieren a definiciones diferentes del PIB. En particular, la metodología posterior incluye en el PIB actividades que no se incluían en la metodología anterior y que mecánicamente disminuyen la participación del PIB industrial en el PIB total" (Carranza y Moreno, 2013:1-18)

Pero como las diferencias, en general, no son tan protuberantes, las tendencias observadas si son válidas. Como puede verse al final de esta sección, la PB creció durante los 45 años a una tasa anual acumulativa del 5\% y el PIB al 4,4\%. En el caso del PIB, coincide con la tasa observada en períodos más extensos, que incluyen este mismo lapso. Tasa de crecimiento económico bruto (PIB total) que, si bien no es excepcional, tampoco es muy baja, dado que es acumulativa para un período que no es tan corto. Incluso si miramos el crecimiento económico neto (PIB per cápita) tampoco es decepcionante, pues como puede verse en la sección $\mathrm{C}$ de la tabla 1 para los 45 años fue del $2,32 \%^{8}$. Pero a pesar de este crecimiento económico no mejoran en proporción similar las condiciones de vida de la inmensa mayoría de la población colombiana.

$\mathrm{Al}$ respecto puede recordarse que en 1958, hace más de medio siglo, la misión Economía y Humanismo, dirigida por el sacerdote Louis Joseph Lebret (1897-1966) estimaba que si en un lapso de 25 años se lograra un crecimiento del producto bruto en especie, esto es, entendido como una canasta de bienes y servicios en valores constantes, del 4,5\% anual, tasa denominada en el texto como norma de lo posible, el país alcanzaría lo que llamaba un desarrollo integral armónico. En

8. Para el período 1923-2010 (88 años) la tasa geométrica de crecimiento del PIB (crecimiento bruto) fue del 4,49\% anual, igual que la observada en el lapso 1959-2010, que corresponde al periodo analizado en la investigación institucional Colombia: Principales transformaciones económicas, sociales y políticas que se adelanta en el Observatorio sobre Desarrollo Humano de la Universidad Autónoma de Colombia, pero la tendencia es que el crecimiento económico pierde ritmo, lo que es grave a mediano plazo; para el período 1959-1978 (gobiernos de Alberto Lleras Camargo a Alfonso López Michelsen) la tasa fue de 5,66\%; luego, de 1979 a 1990 (gobiernos de Turbay Ayala a Barco Vargas) disminuyó a 4,30\% y en 1991-2010 (gobiernos de Gaviria Trujillo a la segunda administración Uribe Vélez) «cayó» al 3,33\%. La tasa de crecimiento neto (PIB per cápita) fue del 2,26\% para 1959-2010. Véase SILVA-COLMENARES, Julio. Colombia: Crecimiento económico y desarrollo humano. Algunas características del proceso en el lapso 1959-2010, En: Varios, Globalización, crecimiento y desarrollo, Bogotá: Academia Colombiana de Ciencias Económicas, 2013, tabla 1, p. 143. 
términos concretos proponía lo siguiente: "Si se adoptan las medidas adecuadas, dadas las potencialidades colombianas (...), no es imposible alcanzar este objetivo, aunque sea muy superior a la elevación media de los países desarrollados, estimada en algo menos del $2 \%$. Pero un país que comienza a desarrollarse y dispone de grandes recursos naturales aun no explotados, o mal explotados, puede sobrepasar considerablemente esta tasa de crecimiento, como lo ha demostrado el ejemplo ruso. Una planeación correctamente establecida, que evite todo desperdicio de consumo y todo error en los equipos necesarios y enérgicamente ejecutada, puede lograrlo" (Misión Economía y Humanismo, 1958: 109).

Como lo vimos más atrás, Colombia tuvo un crecimiento económico bruto durante los 45 años que cubre este análisis del 4,42\%, cumpliendo casi con exactitud la norma de lo posible que proponía la Misión Lebret, pero las condiciones de vida de la inmensa mayoría de los colombianos no han avanzado hacia el anhelado desarrollo integral armónico ${ }^{9}$. Por tanto, nuestro preocupante atraso en el desarrollo humano no tiene una razón explicativa en el crecimiento económico, sino en el modo de desarrollo que hemos «padecido», el que ha sido cerrado, excluyente y oligárquico. Por eso, en otros documentos estamos proponiendo, como «utopía posible», un nuevo modo de desarrollo humano, para la realización de la libertad y la búsqueda de la felicidad. (Silva-Colmenares, 2013:143).

Volviendo al tema central de estas páginas, en la sección C de la tabla 1 se observa que no sólo hubo un crecimiento económico neto significativo en los 45 años [2,87\% en la PB y 2,32\% en el PIB, ambos como tasa anual acumulativa per cápita], sino que la productividad bruta también aumentó -dado el mayor ritmo de crecimiento de la producción respecto al incremento de la población-, pues la PB por persona ocupada se multiplicó por 2,4 veces en el lapso analizado, o sea a una tasa anual acumulativa de 2,01\%, y el PIB por persona ocupada se multiplicó por 1,9 veces, es decir, aumentó a una tasa acumulativa del 1,46\% anual; ambos indicadores calculados sobre pesos de 1994. Como era de esperarse, estos ritmos fueron superiores en la producción material [4 veces en los 45 años o 3,12\% anual en la PB y 2,8 veces o $2,34 \%$ anual en el PIB] que en las actividades de servicio $[1,14 \%$ y $0,58 \%]$, lo que es perjudicial a largo plazo, pues son las actividades económicas con mayor crecimiento.

Para entender mejor por qué la industria manufacturera tiene un mayor peso relativo en la PB que en el PIB, basta observar en la sección D de la tabla 1 que es el sector que muestra la mayor relación entre PIB y PB, al tiempo que esa relación ha aumentado en los 45 años para todos los sectores, excepto para servicios financieros y de alquiler de vivienda. Esto indica que cada vez en el acumulado final de un sector se incorporan más productos y servicios provenientes de otros sectores, o sea, en términos generales, más consumo intermedio, que puede ser de origen nacional o importado. Mientras en el total (renglón 12) aumentó de 1,6 veces en

9. Véase al respecto la parte C. El «vaso medio vacío» del desarrollo humano, en Julio Silva-Colmenares. Colombia: Crecimiento económico y desarrollo humano. Algunas características del proceso en el lapso 1959-2010, En: Varios, Globalización, crecimiento y desarrollo, Bogotá: Academia Colombiana de Ciencias Económicas, 2013, pp. 111 a 139. 
1965 a 2,0 en 2010, esto es, un 27,5\%, con una ligera disminución en 1978 y 1990, en la industria manufacturera también aumentó de 2,9 veces en 1965 a 3,8 en 2010 (renglón 3), o sea un 32,4\%, con disminución para 1978 y 1990. Es decir, por cada $\$$ en el PIB, en la práctica valor agregado, se incorporan $\$ 3,8$ provenientes de otros sectores, cuando en 1965 eran 2,90 pesos, medidas las unidades monetarias en pesos constantes de 1994. Luego siguen con mayor consumo intermedio relativo, en términos generales, los servicios personales, a las empresas, de gobierno y doméstico, así como transporte, almacenamiento y comunicaciones y la construcción y obras públicas. En promedio, en la producción material se incorpora casi un $50 \%$ más consumo intermedio que en los servicios, pues mientras en la primera la relación es de 2,55, en la segunda es de 1,71 para el año 2010.

\section{EL RITMO DESIGUAL DE CRECIMIENTO ECONÓMICO Y LOS CAMBIOS EN LA COMPOSICIÓN SECTORIAL}

Una de las características de las economías modernas es el crecimiento económico desigual, no sólo entre zonas geográficas, ya sea a nivel de continentes o de países, sino entre sectores o actividades, lo que produce con frecuencia desequilibrios críticos, con efectos a veces muy perjudiciales para el desarrollo humano, esto es, para las condiciones en que viven los seres humanos. Aunque no puede ser claro en el primer momento, la disminución en el peso relativo de la industria, tanto en el PIB como en la PB, se dio a pesar de que mantuvo un ritmo de crecimiento positivo, aunque sin llegar a los niveles de otros países ni a la expansión y diversificación deseadas.

Para corroborar que el comportamiento de la industria manufacturera no fue tan negativo como lo podría indicar su pérdida de peso relativo, en la tabla 2 puede observarse que el incremento en el CI y en el VA, medido en términos per cápita reales, fue de $2,1 \%$ y $1,9 \%$ anuales, mientras en los cinco sectores que conforman la llamada «economía real» tales cifras fueron de 2,6\% y 2\%, si bien estos guarismos están afectados por el débil desempeño del sector agropecuario (1,7\% y -0,4\%). No obstante, es preocupante que la industria crezca a menor ritmo que muchas otras actividades, ya que siempre tiene un gran efecto multiplicador por la demanda que genera a otros sectores. Ya observamos antes, en la sección D de la tabla 1, que la industria muestra la más alta relación entre PB y PIB, lo que corrobora la amplia demanda a otros sectores.

Como es obvio, en la tabla 2 se destacan los incrementos per cápita de electricidad, gas y agua y de construcción y obras públicas, lo que confirma el incremento que hubo en la disponibilidad de estos bienes y servicios, aunque todo indica que ha debido ser mayor y de mejor calidad. Para el conjunto de la economía, el VA per cápita aumentó de 821 mil \$ de 1994 en 1965 a casi 2,7 millones en 2010, más de 3 veces, en términos reales, lo que indica un incremento anual acumulable de $2,7 \%$, superior al incremento de la población, aunque inferior al crecimiento de la población ocupada que, en cifras globales, osciló entre 2,9\% y 3,1\%.

La «caída» en las actividades agropecuarias debe analizarse con detenimiento, pues por condiciones naturales el país debe utilizar más y mejor el potencial productivo del campo, para sostener no sólo las necesidades de la creciente urbanización 
sino de la demanda en aumento de alimentos y materias primas agropecuarias por el resto del mundo. Además, la disminución relativa del PIB agropecuario debe estar afectando el ingreso de los campesinos y los trabajadores rurales, pues la remuneración al trabajo es uno de sus componentes fundamentales.

Como destaca un conocido texto de Historia económica de Colombia, en la transformación estructural ocurrida en la composición del PIB el "gran ganador fue el sector minero, que había ocupado un papel más bien secundario en la economía nacional desde comienzos del siglo XX. Esto se asocia a los cambios en la política de hidrocarburos de 1974, los importantes descubrimientos de reservas de petróleo y gas que le sucedieron y el desarrollo de grandes proyectos estatales, en asocio con multinacionales, en los sectores de níquel y carbón. El sector aurífero, la actividad minera con raíces más profundas en la historia, también se recuperó desde los años setenta, cuando se abandonó la paridad fija entre el dólar y el oro”. Como se lee a continuación en esa Historia el "gran perdedor fue el sector más dinámico de la fase anterior de desarrollo: la industria manufacturera. El estancamiento en la participación de la industria en el PIB en la segunda parte de los años setenta fue sucedido en las dos siguientes y, particularmente, en los años noventa, por un abierto proceso de desindustrialización" (Ocampo, Romero y Parra, 2007:348). Si bien -creemos nosotros- no podría hablarse de un comprobado proceso de «desindustrialización», pues la producción material o en valores de uso ha aumentado, como lo muestra la diversificación y ampliación de la oferta de productos, la industria debe recuperar el ritmo perdido, para mejorar el suministro al mercado interno y buscar nuevos «nichos» en el exterior.

Como decíamos en 1985 con base en lo que veíamos en ese momento -adelantándonos, por desgracia, a lo que ocurrió en la realidad-, la "reorientación en el proceso de industrialización incidirá de manera negativa sobre la estructura industrial existente. Al abrirse las importaciones y «promocionarse» las exportaciones se agotará la actual recuperación y se acelerará la presencia de la crisis en aquellas ramas que dependen más del mercado interno y no tienen la suficiente capacidad de competencia con las transnacionales. (...)" (Silva-Colmenares, 1985:95). Asimismo, un análisis más específico sobre la evolución de la industria manufacturera en los años setenta y ochenta del siglo pasado hablaba ya de que la industria colombiana tenía un menor peso relativo que en América Latina y en los países llamados de «industrialización reciente» ${ }^{\mathbf{1 0}}$, debido a una baja competitividad. Como se lee allí, en "relación con este último aspecto, los resultados de un trabajo reciente muestran que mientras entre 1930 y 1974 la contribución de los cambios técnicos al crecimiento del valor agregado industrial osciló alrededor de 50\%, entre 1974 y 1980 cayó sólo a 9\%. (...)” (Silva-Colmenares, 1985:95).

Otro aporte al respecto lo da el ex ministro Rodrigo Botero Montoya, quien en un artículo señalaba que el "PIB industrial pasó de US\$8.806 millones en 1991 a US\$36.688 millones en 2010", lo que implica un aumento en $317 \%$ durante este lapso; pero si hacemos el cálculo sobre valores en pesos colombianos constantes de 1994, como es la unidad de medida monetaria que utilizamos nosotros, tal

10. En esos países la industria representaba en 1985 el 38\% del PIB, en promedio. 
incremento se mantiene positivo pero se reduce a 23,3\% entre el año final (1990) del gobierno Barco Vargas y el último año (2010) de la segunda administración Uribe Vélez ${ }^{11}$. Y después de mencionar cifras también crecientes de importaciones y exportaciones por parte de la industria, concluía Botero Montoya que estos "indicadores no revelan síntomas de retroceso industrial".

Para darle más peso a su percepción, Botero Montoya decía que había que tener en cuenta "el reemplazo del modelo de sustitución de importaciones en una economía cerrada, enfocada hacia el mercado interno [nota de JS-C: pero no estaba tan enfocada al mercado interno, pues el consumo de los hogares perdió peso relativo en el PIB, como lo comprobamos en otro trabajo [Silva-Colmenares)] por el modelo de promoción de exportaciones, enfocado hacia el mercado mundial, gracias a una economía abierta al comercio internacional" [nota de JS-C: tampoco tanto, como lo hemos mostrado]. En cambio, coincidimos, en lo fundamental, con Botero cuando señala que el "esquema de economía cerrada estimula el surgimiento de los denominados molinos de aranceles, dependientes de la protección estatal, una forma ficticia de industrialización de escasa utilidad social. A determinadas empresas se les permite obtener rentas explotando un mercado interno cautivo, obligado a absorber productos de baja calidad y altos precios” (Botero, 2011).

Volviendo a la llamada «desindustrialización», el incremento de la productividad debe ser un factor explicativo de la disminución del peso relativo de la industria y otras actividades de producción material dentro del PIB total, pues supone más unidades de producto por unidad de tiempo remunerada ${ }^{12}$, mientras que la desaparición progresiva del «paraguas» de un Estado privilegiativo obligó a muchos empresarios a reducir costos para buscar precios relativos más competitivos, lo que también implica una disminución del valor total de la producción, aunque puede haber aumentado su volumen y expresión en valores de uso.

También un documento reciente del Dpto. Administrativo Nacional de Estadística -DANE-, que compara el nuevo Sistema de Cuentas Nacionales con base 2005 con el anterior (basado en el año 2000), resalta algunos resultados significativos que explican en parte la «caída» de las actividades agropecuarias y manufactureras en el PIB, aunque debe haber otras causas que hay que investigar con más detalle. Entre éstos destaca el incremento del coeficiente técnico del consumo intermedio, esto es, la relación que representa el consumo intermedio respecto a la producción bruta (CI/PB), como lo hemos mostrado de manera resumida en párrafos anteriores. En el caso del sector agropecuario, de seis actividades que lista el documento, en cinco aumentó este coeficiente. En la industria, de 27 ramas manufactureras, en 15 aumentó tal coeficiente, siendo la mayoría de las que tienen los procesos de

11. Como puede verse en la sección B de la tabla 1, el PIB de la industria manufacturera pasó de 11,3 billones en 1990 a 14 billones en 2010, a precios constantes de 1994.

12. Recuérdese que la remuneración al trabajo es un importante componente del PIB, entendido como una sumatoria de valores agregados; todas las formas de remuneración al trabajo y al capital constituyen, en lo fundamental, el PIB de cualquier rama. En el documento del DANE que se cita más adelante, en la nueva base de Cuentas Nacionales año 2005 el valor agregado representa el 91,5\% del PIB total; el 8,5\% restante corresponde a impuestos menos subvenciones sobre los productos (página 100). 
producción más complejos, como tejidos y confecciones, papel y cartón, productos químicos, de caucho y plástico, productos de minerales no metálicos y de la metalurgia básica, así como fabricación de maquinaria y equipo) ${ }^{\mathbf{1 3}}$. Como es natural, tal incremento lleva a una disminución relativa del valor agregado en las actividades agropecuarias e industriales, o sea en su PIB.

Igual ocurre con las importaciones, pues mientras mayor sea la proporción del componente importado en el consumo intermedio y menor la necesidad de producción interna, más se afecta, en términos negativos, el valor agregado. En este sentido, es pertinente lo que dicen Ocampo y colaboradores: "En el contexto del auge minero, esta crisis industrial y agropecuaria sugiere que la reestructuración productiva que experimentó el país estuvo asociada, al menos en parte, a lo que en la literatura económica internacional se conoce como «enfermedad holandesa»"(Ocampo, Romero y Parra, 2007: 348) Es decir, el país tuvo recursos externos para pagar la condición de importador neto, como lo mostramos en otro trabajo ya citado (Silva-Colmenares, 2013: 105-108).

Como señalamos en párrafos anteriores, muchas actividades de servicio a la producción, como aseo, mantenimiento, labores de contabilidad y ventas, producción de algunos insumos y otras similares, que ahora las realizan empresas especializadas, ya no cuentan como valor agregado sino como consumo intermedio, lo que también explica su pérdida de peso relativo. El valor agregado se trasladó a una rama de los servicios. Un estudio de investigadores del Banco de la República, ya citado, muestra que "de 1994 a 2005 estos servicios por si solos pasaron de ser $3,2 \%$ a $9,9 \%$ de las compras intermedias de la industria”. Este mismo estudio mostró evidencia "de que la industria manufacturera ha desintegrado en alguna medida sus actividades productivas. Esta observación es consistente con diversas teorías de la organización de la firma que describen las circunstancias bajo las cuales las firmas prefieren proveerse servicios a través del mercado en vez de producirlos internamente. La determinación del mecanismo o los mecanismos precisos que generan estos incentivos es un asunto que queda pendiente en nuestra agenda investigativa" (Carranza y Moreno: 15-18).

$\mathrm{El}$ incremento en el peso relativo de los establecimientos bancarios y similares muestra no tanto la existencia de una vigorosa «red» de instituciones que prestan un servicio eficiente-como es más evidente en los países ricos-, sino el efecto de unas abultadas ganancias de monopolio, que crecen quizá a mayor ritmo que en otros sectores, por lo que aumenta el valor agregado y, por consiguiente, su peso relativo en el PIB. Algo similar puede suceder con los servicios sociales y personales, en donde una excesiva burocracia y baja productividad esté llevando a un incremento exagerado de la remuneración global al trabajo, lo que también aumenta el valor agregado, sin que la sociedad se beneficie mucho de ello. Estas, y otras cuestiones, como lo ocurrido en la industria, son deformaciones que distorsionan la medición del PIB y que deben investigarse con más detenimiento.

13. DANE. Principales cambios metodológicos y resultados. Bogotá, noviembre de 2010, pp. 105 a 107, tomado de www.dane.gov.co_el 12 de julio de 2011 


\section{A MANERA DE CONCLUSIÓN}

Este sucinto análisis permite retomar lo planteado en el primer párrafo de la nota: Para hablar de desindustrialización no es suficiente tomar como medida la participación en el PIB, o en el empleo, por lo que habría que adelantar una investigación más compleja, que cubra otros aspectos, y que tenga en cuenta los cambios estructurales ocurridos en las últimas décadas en la transformación de bienes anteriores en nuevos valores de uso, lo que cobija hoy a sectores distintos a la industria manufacturera, por lo que la idea de industria y el concepto de industrialización también tendrían que ampliarse o modificarse. Es decir, habría que tener en cuenta los cambios sustanciales que han ocurrido en el proceso de trabajo. No solo ha cambiado en dónde se produce (localización) y cómo se produce (se ha fraccionado la cadena de producción, incluida la ubicación geográfica y el tipo de empresa, y se ha reducido el tiempo de trabajo por unidad de producto), sino con quién se produce (se sustituyen trabajadores manuales por trabajadores intelectuales) y con qué se produce (se sustituyen materiales antiguos, pesados, por materiales nuevos, livianos, con «miniaturización»y «robotización» de los productos, lo que implica menor esfuerzo físico). Todo esto ha elevado la productividad por unidad de tiempo, lo que reduce el empleo sin que disminuya la cantidad producida, y ha reducido los costos unitarios, lo que se refleja en disminución de los precios finales, facilitándose el aumento del consumo. Todo esto ayudaría a entender la paradoja de la «desindustrialización» con que se inicia este escrito: Tenemos cada vez más bienes industriales, que incorporan el más reciente avance tecnológico, lo que hace que disminuyan los costos y los precios individuales y, por consiguiente, el valor de la producción industrial puede representar una menor porción de la producción total.

Para finalizar estas notas, recordemos que la preocupación por la calidad de los resultados que se desprenden de la medición de variables macroeconómicas, como el PIB y otras similares, ya empezó a expresarse desde mediados del siglo pasado. Autores como el economista ruso-estadounidense y premio Nobel de Economía de 1973 WassilyLeontief (1906-1999), el holandés JanTibergen (1903-1994), premio Nobel de 1969, el estadounidense Paul Samuelson (1915-2009), el economista húngaro Peter Thomas Bauer (1915-2002), la economista inglesa Joan Robinson (1903-1983) y el profesor germano-británico Ernest F. Schumacher (1911-1977), entre otros, ya se habían manifestado sobre lo que mide y no mide el PIB, y otras variables agregadas, y las interpretaciones que pueden tener sus resultados (SilvaColmenares, 2013: 110 y ss.).

Como la discusión al respecto continúa, en febrero de 2008 el presidente de Francia en ese momento, Nicolás Sarkozy, creó,con el auspicio de su gobierno, un grupo de trabajo bajo la orientación de los profesores Joseph Stiglitz, AmartyaSen y Jean Paul Fitoussi y conocido, por su denominación en francés, como la Commission sur la Mesure de la Performance Économique et du Progrès Social, aunque en los medios de comunicación se le conoció más como la Comisión Sarkozy. (Comission on the Measurement of Economic Performance and Social Progress, en 
inglés, y Comisión sobre la Medición del Desarrollo Económico y del Progreso Social, en español). El argumento fundamental del presidente Sarkozy para proponer la Comisión fue la insatisfacción que existía en muchos medios gubernamentales y académicos sobre la aplicación y resultados de la información estadística referente a la actividad económica y la vida de la sociedad, en especial.

Aunque la Comisión se dedicó de manera preferencial a analizar los problemas de la medición del desarrollo, también le dedicó espacio al crecimiento y a dilucidar lo que expresa en si el PIB. Puede decirse que el Informe Final se centra en tres ideas fundamentales: a) el ser humano, pero en especial, los hogares, deben ser el centro de la reflexión y el análisis; b) hay que valorar con una perspectiva nueva la relación entre el Estado y los hogares, sobre todo el efecto de las transferencias gubernamentales, y c) recuperar criterios más precisos y pertinentes, tanto cuantitativos como cualitativos, para medir mejor, y con las diferencias necesarias, las actividades económicas y las condiciones de vida de las personas.

El primer párrafo de la Síntesis del Informe Final destaca que la Comisión tuvo la "misión de determinar los límites del PIB [Producto Interno Bruto] como indicador de los resultados económicos y del progreso social, reexaminar los problemas relativos a la medición, identificar datos adicionales que podrían ser necesarios para obtener indicadores de progreso social más pertinentes, evaluar la viabilidad de nuevos instrumentos de medición y debatir sobre una presentación adecuada de datos estadísticos"14. La Comisión acepta que "la crisis actual nos brinda una lección muy importante: quienes se esfuerzan por guiar nuestras economías y nuestras sociedades son como pilotos que pretenden mantener el rumbo sin una brújula fiable”. Por eso afirma que el Informe se dirige, en primer lugar, a los responsables políticos. En segundo lugar está destinado a las autoridades que desean tener indicadores más adecuados para concebir, aplicar y evaluar políticas, y en tercer lugar a la comunidad académica, de la que se espera que "se muestre más prudente a la hora de confiar en determinadas estadísticas...”.

En este sentido, llama la atención sobre el hecho de que es "posible que los conceptos estadísticos sean adecuados, pero que el proceso de medición sea imperfecto", al tiempo que reconoce que "existe un debate sobre la elección de los conceptos pertinentes y el uso adecuado de los conceptos". Y a continuación plantea que es "posible que las estadísticas empleadas habitualmente no reflejen determinados fenómenos que influyen cada vez más en el bienestar de los ciudadanos". Por tanto, una de sus primeras conclusiones es que no es erróneo, en sí, el cálculo del PIB, "sino que se emplea de forma errónea”. Por ejemplo, cómo cuando el dato del PIB per cápita se convierte en sinónimo de medición del desarrollo, como hemos

14. Ésta, y otras referencias textuales, están tomadas de la Síntesis y Recomendaciones (17 páginas) publicada en español. La versión completa del Informe Final tiene 291 páginas, pero sólo hay versiones disponibles en inglés y francés. El texto de la Síntesis fue «bajado» en enero de 2010 de la página de Internet de la embajada de Francia en Argentina (www.embafrancia-argentina.org. El Informe Final puede verse en www.stiglitz-senfitoussi.fr 
venido insistiendo un grupo creciente de investigadores, porque sigue siendo una medida que relaciona oferta acumulada de bienes y servicios con un conglomerado de personas, pero no dice nada sobre cómo se utiliza esa oferta por las personas, en sí. Algo similar puede estar pasando con la medición de la actividad industrial, como se ha querido plantear en esta nota. Como dice el Informe, "ha llegado la hora de que nuestro sistema estadístico se centre más en la medición del bienestar de la población que en la medición de la producción económica (...)”. Sin dejar de medir la oferta, hay que darle más importancia al análisis de la demanda, sobre todo de los hogares, porque por esa vía es más fácil ver las reales condiciones de vida de una población.

Si cambia de manera sustancial el proceso de trabajo habría que cambiar el método con que se miden los diferentes pasos, hasta llegar al producto final. $\mathrm{O}$ sea, el PIB ya no sería la mejor medida de la actividad económica. Por tanto, como producto de esta comparación proponemos discutir, como lo dijimos en las páginas iníciales de esta nota, si no es conveniente tener dos variables macroeconómicas que sean simultáneas y complementarias: la PB, como materialización de la actividad económica, y el PIB como expresión de la riqueza neta creada en bienes y servicios expresados en unidades monetarias, en lo fundamental como sumatoria de valores agregados, pues existen bienes y servicios que no tienen expresión monetaria y para los cuales es necesario diseñar nuevos métodos de medición.

Como se ve, no será fácil, pero tampoco imposible, hacer la distinción entre crecimiento y desarrollo, ya que se ha avanzado bastante al respecto, y desde hace más de medio siglo. De otro lado, como lo vimos en párrafos anteriores, sin abandonar la medición del crecimiento económico, esto es, la disponibilidad u oferta de bienes y servicios para satisfacer necesidades, y más bien «ampliándola», para incluir bienes y servicios que hoy son «invisibles», hay que mejorar la medición del desarrollo humano, para identificar el nivel de vida alcanzado por una sociedad determinada y precisar los pasos necesarios para avanzar hacia condiciones más dignas de bien-estar, incluyendo aspectos nuevos como la realización de la libertad y la búsqueda de la felicidad. 


\section{REFERENCIAS}

ASOCIACIÓN DE ACADEMIAS DE LA LENGUA ESPAÑOLA (2010). Nueva gramática de la lengua española. Manual. Bogotá, Editorial Planeta Colombia.

BOTERO MONTOYA, Rodrigo (2011). Añoranzas proteccionistas. En: La República, 2-3 de abril de 2011, p. 39.

CLAVIJO, Sergio; VERA, Alejandro y FANDIÑO, Alejandro (2012). La desindustrialización en Colombia. Análisis cuantitativo de sus determinantes. Anif, Bogotá.

CARRANZA, Juan Esteban y MORENO, Stefany (2013) Tamaño y estructura vertical de la cadena de producción industrial colombiana desde 1990. Borradores de Economía: Banco de la República.

DANE (2010). Principales cambios metodológicos y resultados. Bogotá, noviembre, pp. 105 a 107, tomado de www.dane.gov.co_el 12 de julio de 2011.

EMBAJADA DE ARGENTINA (2010). (www.embafrancia-argentina.org. El Informe Final puede verse en www.stiglitz-sen-fitoussi.fr

ECHAVARRÍA, Juan José y VILLAMIZAR, Mauricio (Con la colaboración de Juanita González) (2006). El proceso colombiano de desindustrialización. Borradores de economía: Banco de la República, enero, No. 36.

FERREIRA SEQUEDA María Teresa (2010). La precarización del trabajo en la ciudad como una de las nuevas formas de empobrecimiento en Colombia. Bogotá: Academia Colombiana de Ciencias Económicas.

MISIÓN ECONOMÍA Y HUMANISMO (1958). Estudio sobre las condiciones del desarrollo de Colombia, Bogotá: Presidencia de la República.

OCAMPO GAVIRIA, José Antonio; ROMERO BAQUERO, Carmen Astrid y PARRA, María Ángela (2007). La búsqueda, larga e inconclusa, de un nuevo modelo (1981-2006), En: OCAMPO GAVIRIA, José Antonio. Comp. Historia económica de Colombia, Bogotá: Planeta-Fedesarrollo.

SILVA-COLMENARES, Julio y PADILLA PARDO, Carolina (2013). Transnacionalización del grupo financiero Suramericana. En: revista Economía y Desarrollo, Universidad Autónoma de Colombia, marzo 2013. Vol. 12 , No. 1 , pp. 29-57.

SILVA-COLMENARES, Julio (2013). Colombia: Crecimiento económico y desarrollo humano. Algunas características del proceso en el lapso 1959-2010, En: Varios, Globalización, crecimiento y desarrollo, Bogotá: Academia Colombiana de Ciencias Económicas.

SILVA-COLMENARES, Julio (1985). El plan de ajuste impuesto por el Fondo Monetario Internacional a Colombia y algunas incidencias económicas, sociales y políticas, En: Varios. El trasfondo-monetarismo del ajuste. Bogotá: Universidad Autónoma de Colombia.

SILVA-COLMENARES, Julio (2013) Nuevo modo de desarrollo. Una utopía posible, Bogotá: Ediciones Aurora-Universidad Autónoma de Colombia.

UNIVERSIDAD AUTÓNOMA DE COLOMBIA (2013). Observatorio sobre desarrollo humano. 
Revista TENDENCIAS Vol. XV No.

COLOMBIA: COMPOSICIÓN SECTORIAL DE LAPRODUCCIÓN BRUTA -PB-Y EL PRODUCTO INTERNO BRUTO -PIB$1965,1978,1990$ y 2010

\begin{tabular}{|c|c|c|c|c|c|c|c|c|c|c|}
\hline \multirow{3}{*}{ Sectores/Años } & \multicolumn{2}{|c|}{1965} & \multicolumn{2}{|c|}{1978} & \multicolumn{2}{|c|}{1990} & \multicolumn{2}{|c|}{2010} & \multirow{2}{*}{\multicolumn{2}{|c|}{$\begin{array}{c}\text { Cambio \% en el } \\
\text { peso relativo } \\
2010 / 1965\end{array}$}} \\
\hline & \multirow[t]{2}{*}{ PB } & \multirow[t]{2}{*}{ PIB } & \multirow[t]{2}{*}{ PB } & \multirow[t]{2}{*}{ PIB } & \multirow[t]{2}{*}{ PB } & \multirow[t]{2}{*}{ PIB } & \multirow[t]{2}{*}{ PB } & PIB & & \\
\hline & & & & & & & & & PB $y$ & PIB \\
\hline & & A- COMP & OSICIÓN F & PORCENT & TUAL & & & & & \\
\hline 1 Agricultura, silvicultura, caza y pesca & $19,4 \%$ & $27,3 \%$ & $16,4 \%$ & $23,0 \%$ & $12,5 \%$ & $16,2 \%$ & $5,6 \%$ & $6,5 \%$ & $-71,1 \%$ & $-76,2 \%$ \\
\hline 2 Mineria & $3,6 \%$ & $4,5 \%$ & $1,2 \%$ & $1,3 \%$ & $6,7 \%$ & $9,3 \%$ & $6,3 \%$ & $8,4 \%$ & $75,9 \%$ & $88,8 \%$ \\
\hline 3 Industria manufacturera & $34,4 \%$ & $19,0 \%$ & $38,0 \%$ & $23,2 \%$ & $34,7 \%$ & $19.9 \%$ & $24,0 \%$ & $12,8 \%$ & $-30,3 \%$ & $-32,7 \%$ \\
\hline 4 Electricidad, gas y agua & $0,8 \%$ & $0,9 \%$ & $1.1 \%$ & $1,3 \%$ & $2,1 \%$ & $2,5 \%$ & $3,5 \%$ & $3,6 \%$ & $318,3 \%$ & $282,2 \%$ \\
\hline 5 Construcción y obras públicas & $3,7 \%$ & $3,0 \%$ & $4,6 \%$ & $4,1 \%$ & $4,9 \%$ & $4,9 \%$ & $8,8 \%$ & $7,2 \%$ & $135,2 \%$ & $140,3 \%$ \\
\hline 6 Subtotal prod. material (Reng. 1 a 5 ) & $62,0 \%$ & $54,7 \%$ & $61,4 \%$ & $53,0 \%$ & $60,9 \%$ & $53,0 \%$ & $48,3 \%$ & $38,5 \%$ & $-22,2 \%$ & $-29,5 \%$ \\
\hline 7 Comercio, reparaciones, hoteles y restaurante & $8,4 \%$ & $13,1 \%$ & $8.7 \%$ & $13,5 \%$ & $8,7 \%$ & $14,1 \%$ & $12,6 \%$ & $11,6 \%$ & $49,7 \%$ & $-11,6 \%$ \\
\hline 8 Transporte, almacenamiento y comunicacione & $6,6 \%$ & $7,1 \%$ & $7,6 \%$ & $8,1 \%$ & $8,5 \%$ & $9,1 \%$ & $8.0 \%$ & $6,4 \%$ & $21,4 \%$ & $-10,4 \%$ \\
\hline 9 Servicios financieros y de alquiler de vivienda & $10,0 \%$ & $14,7 \%$ & $9,4 \%$ & $13,4 \%$ & $8,1 \%$ & $11,1 \%$ & $9,7 \%$ & $19,3 \%$ & $-3,5 \%$ & $31,5 \%$ \\
\hline $\begin{array}{l}10 \text { Servicios personales, a las empresas, de } \\
\text { gobierno y doméstico }\end{array}$ & $12,9 \%$ & $11,6 \%$ & $12,9 \%$ & $12,2 \%$ & $13,8 \%$ & $12,8 \%$ & $21,4 \%$ & $15,8 \%$ & $65,9 \%$ & $36,9 \%$ \\
\hline 11 Otros al & & $-1,1 \%$ & & $-0,3 \%$ & & $-0,2 \%$ & & $8,4 \%$ & & \\
\hline 12 Subtotal servicios (reng. 7 a 11) & $38,0 \%$ & $45,3 \%$ & $38,6 \%$ & $47,0 \%$ & $39,1 \%$ & $47,0 \%$ & $51,7 \%$ & $61,5 \%$ & $36.2 \%$ & $35,6 \%$ \\
\hline Total & $100,0 \%$ & $100,0 \%$ & $100,0 \%$ & $100,0 \%$ & $100.0 \%$ & $100,0 \%$ & $100,0 \%$ & $100,0 \%$ & & \\
\hline B-COMPOS & SICIÓN EN & MILES D & E MILLON & ES DE \$ & DE 1994 & & & & & \\
\hline 1 Agricultura, silvicultura, caza y pesca & $4.848,5$ & $4.265,9$ & $8.646,9$ & $7.718,1$ & $9.963,4$ & $9.228,3$ & $12.520,3$ & $7.131,8$ & & \\
\hline 2 Mnería & 896,0 & 698,4 & 630,9 & 451,8 & $5.345,1$ & $5.298,0$ & $14.086,8$ & $9.251,1$ & & \\
\hline 3 Industria manufacturera & $8.610,5$ & $2.964,8$ & $20.018,6$ & $7.784,6$ & $27.692,9$ & $11.345,7$ & $53.674,2$ & $13.994,9$ & & \\
\hline 4 Electricidad, gas y agua & 211,0 & 147,6 & 598,4 & 421,4 & $1.678,8$ & $1.431,1$ & $7.890,1$ & $3.956,9$ & & \\
\hline 5 Construcción y obras públicas & 935,5 & 469,6 & $2.420,3$ & $1.379,6$ & $3.905,0$ & $2.814,6$ & $19.670,1$ & $7,918,6$ & & \\
\hline 6 Subtotal prod. material (Reng. 1 a 5 ) & $15.501,5$ & $8.546,2$ & $32.315,1$ & $17.755,5$ & $48.585,1$ & $30.117,8$ & $107.841,4$ & $42.253,3$ & & \\
\hline 7 Comercio, reparaciones, hoteles y restaurante & $2.104,8$ & $2.052,2$ & $4.590,8$ & $4.518,1$ & $6.919,5$ & $8.043,0$ & $28.166,4$ & $12.723,3$ & & \\
\hline 8 Transporte, almacenamiento y comunicacione & $1.656,3$ & $1.110,6$ & $3.997,4$ & $2.729,5$ & $6.763,9$ & $5.181,6$ & $17.979,1$ & $6.980,8$ & & \\
\hline 9 Servicios financieros y de alquiler de vivienda & $2.508,8$ & $2.292,5$ & $4.954,9$ & $4,482,7$ & $6.485,9$ & $6.339,1$ & $21.638,7$ & $21.144,8$ & & \\
\hline $\begin{array}{l}10 \text { Servicios personales, a las empresas, de } \\
\text { gobierno y doméstico }\end{array}$ & $3.226,0$ & $1.808,4$ & $6.792,8$ & $4.100,2$ & $11.000,5$ & $7.293,8$ & $47.837,3$ & $17.367,0$ & & \\
\hline 11 Otros a/ & & $-176,3$ & & $-88,1$ & & $-101,4$ & & $9.216,7$ & & \\
\hline 12 Subtotal servicios (reng. 7 a 11 ) & $9.495,8$ & $7.087,5$ & $20.335,8$ & $15.742,4$ & $31,169,8$ & $26.756,1$ & $115.621,6$ & $67.432,7$ & & \\
\hline Total (miles de millones de \$ de 1994) & $24.997,3$ & $15.633,7$ & $52.650,9$ & $33.497,9$ & 79.754 .9 & $56.873,9$ & $223.463,0$ & $109.686,0$ & & \\
\hline TAG de la PB y el PIB, periodo $1965 / 2010$ (45 añ & & & & & & & $4,99 \%$ & $4,42 \%$ & & \\
\hline & C-INDIC & CADORES & SPER CAP & ITA & & & & & & \\
\hline PB y PIB per cápita b/ (miles de \$ de 1994) & 1.374 & 859 & 2.055 & 1.307 & 2.337 & 1.667 & 4.910 & 2.410 & & \\
\hline TAG de la PB y el PIB per cápita, periodo $1965 / 2$ & 010 (45 año & & & & & & $2,87 \%$ & $2,32 \%$ & & \\
\hline $\begin{array}{l}\text { PB y PIB total por persona ocupada c/ (miles } \\
\text { de \$ de 1994) }\end{array}$ & 4.753 & 2.973 & 8.100 & 5.154 & 7.068 & 5.040 & 11.630 & 5.708 & & \\
\hline TAG de la PB y PIB por persona ocupada, period & o $1965 / 201$ & $10(45$ aก๊o & & & & & $2,01 \%$ & $1,46 \%$ & & \\
\hline \begin{tabular}{|l|} 
PB y PIB por persona ocupada, 5 sectores, \\
producción material d/ (miles de \$ de 1994)
\end{tabular} & 4.479 & 2.469 & 8.905 & 4.893 & 11.604 & 7.193 & 17.849 & 6.993 & & \\
\hline TAG de la PB y PIB por persona ocupada, 5 sect & ores, produ & ucción ma & iterial, perio & do $1965 / 2$ & $010(45$ año & & $3,12 \%$ & $2,34 \%$ & & \\
\hline \begin{tabular}{|l|} 
PB y PIB por persona ocupada, servicios u \\
otras actividades e/ (miles de \$ de 1994)
\end{tabular} & 5.281 & 3.942 & 7.083 & 5.483 & 4.392 & 3.770 & 8.777 & 5.119 & & \\
\hline TAG de la PB y PIB por persona ocupada, servic & ios, otras a & ctividades & período 15 & $965 / 2010$ & 45 años) & & $1,14 \%$ & $0,58 \%$ & & \\
\hline D - RELACIÓN & ENTRE PB & B/PIB (ve & ces, sobre & datos en & S de 1994) & & & & & \\
\hline 1 Agricultura, silvicultura, caza y pesca & 1,14 & & 1,1 & & 1,0 & & 1,7 & & & \\
\hline 2 Mneria & 1,28 & & 1,4 & & 1,0 & & 1,5 & & & \\
\hline 3 Industria manufacturera & 2,90 & & 2,5 & & 2,4 & & 3,8 & & & \\
\hline 4 Electricidad, gas y agua & 1.43 & & 1,4 & & 1,1 & & 1,9 & & & \\
\hline 5 Construcción y obras públicas & 1,99 & & 1,7 & & 1,3 & & 2,48 & & & \\
\hline 6 Subtotal prod. material (Reng. 1 a 5) & 1,81 & & 1,8 & & 1,6 & & 2,5 & & & \\
\hline 7 Comercio, reparaciones, hoteles y restaurante & 1,03 & & 1,02 & & 0,8 & & 2,2 & & & \\
\hline 8 Transporte, almacenamiento y comunicacione & $1,4 \mathrm{~S}$ & & 1,46 & & 1,3 & & 2,5 & & & \\
\hline 9 Servicios financieros y de alquiler de vivienda & 1,09 & & 1,1 & & 1,0 & & 1,0 & & & \\
\hline $\begin{array}{l}10 \text { Servicios personales, a las empresas, de } \\
\text { gobierno y doméstico }\end{array}$ & 1,78 & & 1,6 & & 1,5 & & 2,7 & & & \\
\hline 11 Subtotal servicios Reng. 7 a 11) & 1,34 & & 1,2 & & 1,1 & & 1,7 & & & \\
\hline 12 Total & 1,60 & & 1,5 & & 1,4 & & 2,0 & & & \\
\hline & -POBLAC & CIÓN (mil & es de pers & ionas) & & & & & & \\
\hline b/ Población total & 18.15 & & 25.6 & & 34.1 & & 45.5 & & & \\
\hline c/ Población ocupada total & 5.25 & & 6.50 & & 11.2 & & 19.2 & & & \\
\hline d/ Población ocupada en la producción material & 3.46 & & 3.62 & & 4.18 & & 6.04 & & & \\
\hline e/ Población ocupada (resto) & 1.79 & & 2.87 & & 7.05 & & 13.1 & & & \\
\hline a/ En cuanto al Pl8, para los años de 1965,1978 y $19 \mathrm{~s}$ & & & & & & & & & & \\
\hline Para el 2010 corresponde al concep & uestos men & nos Subven & & & & & & & & \\
\hline$P B=$ Producción bruta & $P \mathrm{IB}=$ Produc & cto Interno & bruto & $A G=$ Tasa & anual geome & étrica parae & el periodo ing & dicado & & \\
\hline
\end{tabular}


Julio Silva-Colmenares

En torno a la discusión sobre «Desindustrialización»

Un sucinto análisis respecto a la producción bruta y el Producto Interno Bruto

Tabla 2

PER CÁPITA EN CONSUMO INTERMEDIO Y VALOR AGREGADO - ACTIVIDADES ESCOGIDAS

En miles de \$ de 1994 y TAG para el periodo 1965-2010

\begin{tabular}{|c|c|c|c|c|c|c|c|c|c|c|}
\hline \multirow{2}{*}{ Sectores/Años } & \multicolumn{2}{|c|}{1965} & \multicolumn{2}{|c|}{1978} & \multicolumn{2}{|c|}{1990} & \multicolumn{2}{|c|}{2010} & \multirow{2}{*}{$\begin{array}{c}\text { TAG Clpc } \\
\text { en } 45 \text { años }\end{array}$} & \multirow{2}{*}{$\begin{array}{l}\text { TAG VApc } \\
\text { en } 45 \text { años }\end{array}$} \\
\hline & $\mathrm{Cl}$ & VA & $\mathrm{Cl}$ & VA & $\mathrm{Cl}$ & VA & $\mathrm{Cl}$ & VA & & \\
\hline Población (Miles de habitantes) & \multicolumn{2}{|c|}{18.194} & \multicolumn{2}{|c|}{25.625} & \multicolumn{2}{|c|}{34.124} & \multicolumn{2}{|c|}{45.508} & & \\
\hline - Para el Total (Todos los sectores) & 553 & 821 & 845 & 1.209 & 977 & 1.360 & 2.242 & 2.668 & $3,16 \%$ & $2,65 \%$ \\
\hline - Para el sector agropecuario & 40 & 227 & 53 & 285 & 64 & 228 & 86 & 189 & $1,73 \%$ & $-0,40 \%$ \\
\hline - Para la minería & 12 & 37 & 8 & 17 & 26 & 131 & 64 & 246 & $3,76 \%$ & $4,29 \%$ \\
\hline - Para electricidad, gas y agua & 4 & 8 & 8 & 16 & 14 & 35 & 82 & 91 & $7,10 \%$ & $5,60 \%$ \\
\hline - Para construcción y obras & 26 & 25 & 44 & 51 & 45 & 69 & 222 & 210 & $4,84 \%$ & $4,85 \%$ \\
\hline - Para la industria manufacturera & 316 & 158 & 494 & 287 & 532 & 280 & 808 & 372 & $2,11 \%$ & $1,92 \%$ \\
\hline - Para los 5 sectores anteriores & 398 & 454 & 606 & 655 & 681 & 743 & 1.261 & 1.108 & $2,60 \%$ & $2,00 \%$ \\
\hline
\end{tabular}

- TAG para la población (en 45 años)

$2,06 \%$

$\mathrm{Cl}=$ Consumo intermedio $\quad \mathrm{Clpc}=$ Consumo intermedio per cápita

$\mathrm{VA}=$ Valor agregado $\quad \mathrm{VApc}=$ Valor agregado per cápita

TAG $=$ Tasa anual geométrica 